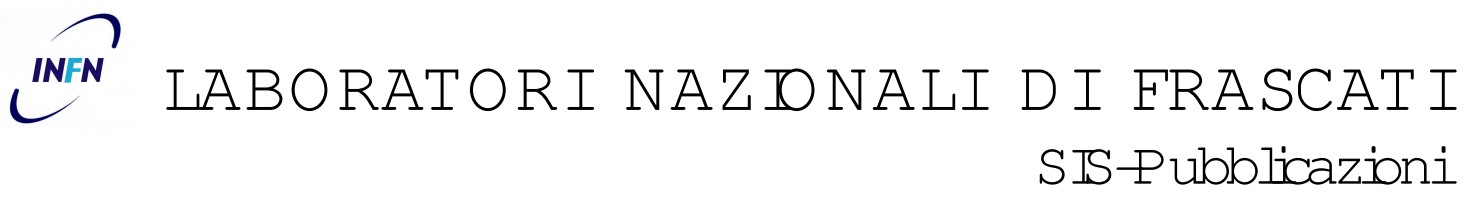

LNF-08/27(P)

November 29,2008

\title{
SENSITIVITY AND ENVIRONMENTAL RESPONSE OF THE CMS RPC GAS GAIN MONITORING SYSTEM
}

\author{
L. Benussi ${ }^{1}$, S. Bianco ${ }^{1}$, S. Colafranceschi ${ }^{1} ; 2 ; 3$, F. L. Fabbri ${ }^{1}$, M. Giardoni ${ }^{1}$ \\ B. Ortenzi ${ }^{1}$, A. Paolozzi, ${ }^{1} ; 2$ L. Passamonti ${ }^{1}$, D. Pierluigi ${ }^{1}$ \\ B. Ponzio ${ }^{1}$, A. Russo ${ }^{1}$, A. Colaleo ${ }^{4}$, F. Loddo ${ }^{4}$, M. Maggi $^{4}$ \\ A. Ranieri ${ }^{4}$, M. Abbrescia ${ }^{4} ; 5$, G. Iaselli $^{4} ; 5$, B. Marangelli ${ }^{4} ; 5$, S. Natali $^{4} ; 5$ \\ S. Nuzzo ${ }^{4}$; 5 , G.Pugliese ${ }^{4}$;5, F. Romano ${ }^{4}$;5, G. Roselli ${ }^{4}$;5, R. Trentadue ${ }^{4}$;5 \\ S. Tupputi ${ }^{4}$;5, R. Guida ${ }^{3}$, G. Polese ${ }^{3}$; 6 , A. Sharma ${ }^{3}$, A. Cimmino ${ }^{7}$;8 \\ D. Lomidze $^{8}$, D. Paolucci ${ }^{8}$, P. Piccolo ${ }^{8}$, P. Baesso $^{9}$, M. Necchi $^{9}$ \\ D. Pagano $^{9}$, S. P. Ratti ${ }^{9}$, P. Vitulo ${ }^{9}$, C. Viviani ${ }^{9}$ \\ 1) INFN Laboratori Nazionali di Frascati, Via E. Fermi 40, I-00044 Frascati, Italy. \\ 2) Università degli Studi di Roma "La Sapienza”, Piazzale A. Moro. \\ 3) CERN CH-1211 Genéve 23 F-01631 Switzerland. \\ 4) INFN Sezione di Bari, Via Amendola, 173I-70126 Bari, Italy. \\ 5) Dipartimento Interateneo di Fisica, Via Amendola, 173I-70126 Bari, Italy. \\ 6) Lappeenranta University of Technology, P.O. Box 20 FI-538 1 Lappeenranta, Finland. \\ 7) INFN Sezione di Napoli, Complesso Universitario di Monte Sant'Angelo, edificio 6, 80126 Napoli, Italy. \\ 8) Università di Napoli Federico II, Complesso Universitario di Monte Sant'Angelo, edificio 6, 80126 Napoli, Italy. \\ 9) INFN Sezione di Pavia, Via Bassi 6, 27100 Pavia, Italy and Università degli studi di Pavia, Via Bassi 6, 27100 Pavia, Italy.
}

\begin{abstract}
Results from the gas gain monitoring (GGM) system for the muon detector using RPC in the CMS experiment at the LHC is presented. The system is designed to provide fast and accurate determination of any shift in the working point of the chambers due to gas mixture changes.
\end{abstract}

PACS: 07.77.Ka; 95.55.Vj; 29.40.Cs

Presented by S. Colafranceschi at the IEEE 08 - 23 October 2008, Dresden, Germany 


\section{Introduction}

Resistive Plate Chambers (RPC) detectors are widely used in HEP experiments for muon detection and triggering at high-energy, high-luminosity hadron colliders, in astroparticle physics experiments for the detection of extended air showers, as well as in medical and imaging applications. At the LHC, muon systems of the CMS experiment rely on Drift Tubes (DT), Cathode Strip Chambers (CSC) and RPCs for their muon trigger system, with a total gas volume of about $50 \mathrm{~m}^{3}$. Utmost attention has to be paid to the possible presence

of gas contaminants which degrade the chamber performance. The gas gain monitoring (GGM) system monitors the gas quality online and is based on small RPC detectors. The working point - gain and efficiency - is continuously monitored along with environmental parameters, such as temperature, pressure and humidity, which are important for the operation of the muon detector system. Design parameters, construction, prototyping and preliminary commissioning results of the CMS RPC Gas Gain Monitoring (GGM) system have been presented previously [1],[7]. In this paper, results on the response of the GGM detectors to environmental changes are presented.

The CMS RPCs are bakelite-based double-gap RPC with strip readout (for construction details see [2] and reference therein) operated with $96.2 \% \mathrm{C}_{2} \mathrm{H}_{2} \mathrm{~F}_{4}-3.5 \%$ Iso- $\mathrm{C}_{4} \mathrm{H}_{10}$ $-0.3 \% \mathrm{SF}_{6}$ gas mixture humidified at about $40 \%$. The large volume of the whole CMS RPC system and the cost of gas used make mandatory the operation of RPC in a closedloop gas system (for a complete description see [3]), in which the gas fluxing the gaps is reused after being purified by a set of filters[4].

The operation of the CMS RPC system is strictly correlated to the ratio between the gas mixture components, and to the presence of pollution due to contaminants that can be be produced inside the gaps during discharges (i.e. HF produced by $\mathrm{SF}_{6}$ or $\mathrm{C}_{2} \mathrm{H}_{2} \mathrm{~F}_{4}$ molecular break-up and further fluorine recombination), accumulated in the closed-loop or by pollution that can be present in the gas piping system (tubes, valves, filters, bubblers, etc.) and flushed into the gaps by the gas flow. The monitoring of the presence of these contaminants, as well as the gas mixture stability, is therefore mandatory to avoid RPC damage and to ensure their correct functionality.

A monitoring system of the RPC working point due to changes of gas composition and pollution must provide a faster and sensitive response than the CMS RPC system itself in order to avoid irreversible damage of the whole system. Such a Gas Gain Monitoring system monitors efficiency and signal charge continuously by means of a cosmic ray telescope based on RPC detectors. In the following will be briefly described the final setup of the GGM system, and the first results obtained during its commissioning at the ISR test area (CERN). 


\section{The Gas Gain Monitoring System}

The GGM system is composed by the same type of RPC used in the CMS detector but of smaller size (2mm Bakelite gaps, $5050 \mathrm{~cm}^{2}$ ). Twelve gaps are arranged in a stack located in the CMS gas area (SGX5 building) in the surface, close to CMS assembly hall (LHC-P5). The choice to install the system in the surface instead of underground allows one to profit from maximum cosmic muon rates. In order to ensure a fast response to working point shifts with a precision of $1 \%, 10^{4}$ events are are required, corresponding to about 30 minutes exposure time on surface, to be compared with a 100-fold lesser rate underground. The trigger is provided by four out of twelve gaps of the stack, while the remaining eight gaps are used to monitor the working point stability.

The eight gaps are arranged in three sub-system: one sub-system (two gaps) is fluxed with the fresh CMS mixture and its output sent to vent. The second sub-system (three gaps) is fluxed with CMS gas coming from the closed-loop gas system and extracted before the gas purifiers, while the third sub-system (three gaps) is operated with CMS gas extracted from the closed-loop extracted after the gas filters. The basic idea is to compare the operation of the three sub-systems and, if some changes are observed, to send a warning to the experiment. In this way, the gas going to and coming from the CMS RPC detector is monitored by using the two gaps fluxed with the fresh mixture as reference gaps. This setup will ensure that pressure, temperature and humidity changes affecting the gaps behavior do cancel out by comparing the response of the three subsystem operating in the same ambient condition.

The monitoring is performed by measuring the charge distributions of each chamber. The eight gaps are operated at different high voltages, fixed for each chamber, in order to monitor the total range of operating modes of the gaps. The operation mode of the RPC changes as a function of the voltage applied. A fraction of the eight gaps will work in pure avalanche mode, while the remaining will be operated in avalanche+streamer mode. Comparison of signal charge distributions and the ratio of the avalanche to streamer components of the ADC provides a monitoring of the stability of working point for changes due to gas mixture variations.

Details on the construction of GGM can be found in [7]. Each chamber of the GGM system consists of a single gap with double sided pad read-out: two copper pads are glued on the two opposite external side of the gap. The signal is read-out by a transformer based circuit A3 (Fig.1). The circuit allows to algebraically subtract the two signal, which have opposite polarities, and to obtain an output signal with subtraction of the coherent noise, with an improvement by about a factor 4 of the signal to noise ratio. The output signals from circuit A3 are sent to a CAEN V965 ADC [6] for charge analysis. 
A typical ADC distribution of a GGM gap is shown in fig.2 for two different effective operating voltage, defined as the high voltage set on the HV power supply corrected for the local atmospheric pressure and temperature. Fig.2 a) corresponding to $\mathrm{HV}_{\text {eff }}=9.9 \mathrm{kV}$ shows a clean avalanche peak well separated from the pedestal. Fig. $2 \mathrm{~b}$ ) shows the charge distribution at $\mathrm{HV}_{\text {eff }}=10.7 \mathrm{kV}$ with two signal regions corresponding to the avalanche and to avalanche+streamer mode.

Fig.3 shows the GGMS single gap efficiency (full dots), and the ratio between the avalanche and the streamer component (open circles), as a function of the effective high voltage. Each point corresponds to a total of 10000 entries in the full ADC spectrum. The efficiency is defined as the ratio between the number of triggers divided by the number of events above 3 ped over ADC pedestal, where ped is the pedestal width. The avalanche to streamer ratio is defined by counting the number of entries in the avalanche (below the ADC threshold (fig. 2 b) and above the pedestal region) and dividing it by the number of streamer events above the avalanche threshold. Both efficiency and avalanche plateau are in good agreement with previous results [5].

In order to determine the sensitivity of GGM gaps to working point shifts, the avalanche to streamer transition was studied by two methods, the charge method and the efficiency method. In the charge method, the mean value of the ADC charge distribution in the whole $\mathrm{ADC}$ range is studied as a function of $\mathrm{HV}_{\text {eff }}$ (fig.4). Each point corresponds to 10000 events in the whole ADC spectrum. In the plot three working point regions are identified

1. inefficiency $\left(\mathrm{HV}_{\text {eff }}<9.7 \mathrm{kV}\right)$;

2. avalanche $\left(9.7 \mathrm{kV}<\mathrm{HV}_{\text {eff }}<10.6 \mathrm{kV}\right.$;

3. avalanche+streamer mode $\left(\mathrm{HV}_{\text {eff }}>10.6 \mathrm{kV}\right)$.

The best sensitivity to working point shifts is achieved in the avalanche+streamer region, estimated to be about $25 \mathrm{ADC} \mathrm{ch} / 10 \mathrm{~V}$ or $1.2 \mathrm{pC} / 10 \mathrm{~V}$.

In the efficiency method, the ADC avalanche event yield is studied as a function of $\mathrm{HV}_{\text {eff }}(5)$. The avalanche signal increases by increasing the $\mathrm{HV}$ applied to the gap, until it reaches a maximum value after which the streamer component starts to increase. The $9.0 \mathrm{kV}-10.0 \mathrm{kV}$ shows a sensitivity to work point changes of approximately $1.3 / \% / 10 \mathrm{~V}$.

\section{Response of GGM to environmental effects}

The workpoint of GGM is affected by environment. However, all environmental effects cancel out thanks to redundancy of the system. Each environmental effect not connected 
with a modification of gas mixture will be cancelled out by a comparison between different RPC chamber flown with the same gas, which are affected by the same environmental parameters.

An example of such cancellation is shown in Fig. 6, where the average charge distribution (black dots) is plotted across a changeover of gas bottles. Data show a sudden increase in the average charge distribution which may interpreted as a shift of working point due to changes in gas mixture composition. By weighing the average charge with a correction factor linearly depending on atmosferic pressure, however, no significant increase is left in the distribution of corrected average charge (green dots) which may signal an anomalous shift due to gas mixture. The cancellation algorithm is applied by correcting variables withing gaps belonging to the same subdetector. Fig. 7 shows the average charge for two chambers working in different regimes at different voltages. The average charge of both chambers is completely correlated, and very well correlated to the atmosferic pressure variations.

\section{Conclusions}

Results from the Gas Gain Monitoring System for the CMS RPC Detector have been reported on. The purpose of GGM is to monitor any shift of the working point of the CMS RPC detector. The GGM is being commissioned at CERN and is planned to start operation by the end of 2008. Preliminary results show good sensitivity to working point changes. The system redundancy allows for effectively cancelling out the environmental effects. Further tests are in progress to determine the sensitivity to gas variations.

\section{References}

[1] M. Abbrescia et al., "Gas analysis and monitoring systems for the RPC detector of CMS at LHC", presented by S.Bianco at the IEEE 2006, San Diego (USA), arXiv:physics/0701014.

[2] R. Adolphi et al. [CMS Collaboration], JINST 3 (2008) S08004

[3] R. Guida et al., " The CMS RPC gas system and the Closed Loop recirculation system”, presented at the RPC Conference, Mumbai, 2008.

[4] G. Saviano et al., "Material, Filter and Gas analysis for the CMS RPC detector in the Closed Loop test setup", presented at the RPC Conference, Mumbai, 2008 to appear on Journal of Instrumentation 
[5] M. Abbrescia et al., "Cosmic ray tests of double-gap resistive plate chambers for the CMS experiment”, Nucl. Instrum. Meth. A 550, 116 (2005).

[6] C.A.E.N. Costruzioni Apparecchiature Elettroniche Nucleari S.p.A. Via Vetraia 11 - 55049 Viareggio (Italy).

[7] L. Benussi et al., "The CMS RPC Gas Gain Monitoring System: an Overview and Preliminary Result", presented in Mumbai08 to appear on Nuclear Instruments and Methods arXiv:0812.1108 [physics.ins-det]. 


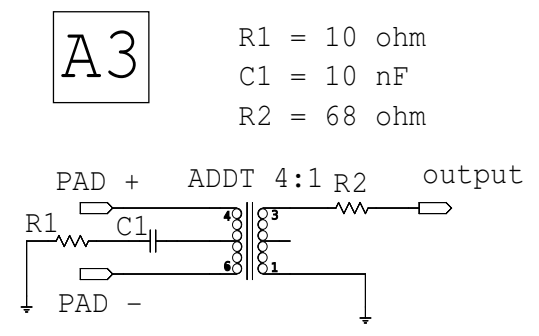

Figure 1: The electric scheme of the read-out circuit providing the algebraic sum of the two pad signal (PAD + and PAD -).

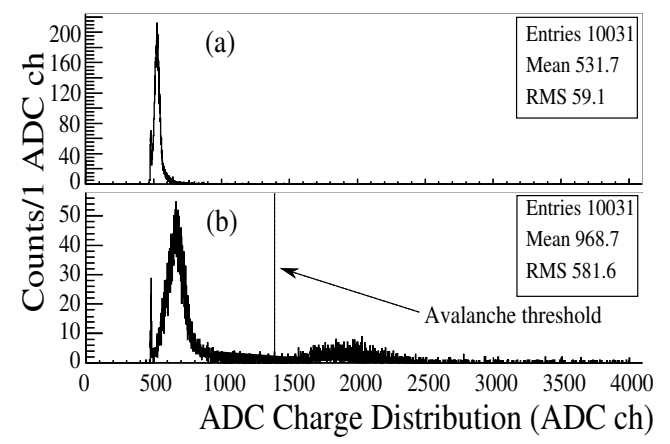

Figure 2: Typical ADC charge distributions of one GGM chamber at two operating voltages. Distribution (a) correspond to $\mathrm{HV}_{\mathrm{eff}}=9.9 \mathrm{kV}$ while distribution (b) to $\mathrm{HV}_{\text {eff }}=10.7 \mathrm{kV}$. In (b) is clearly visible the streamer peak around 1900 ADC channels. The events on the left of the vertical line (1450 ADC channels in this case) are assumed to be pure avalanche events. 


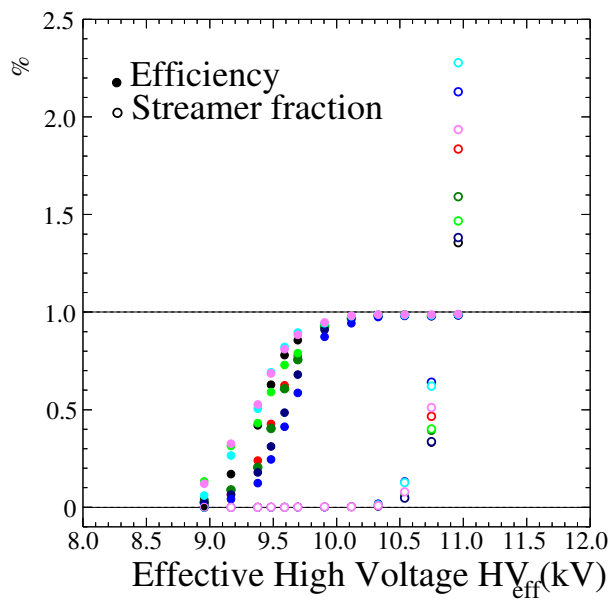

Figure 3: Efficiency plot (full dots) of GGM chambers as a function of $\mathrm{HV}_{\mathrm{eff}}$. The efficiency is defined as the ratio between the number of ADC entries above 3 ped and the number of acquired triggers. Open dot plots correspond to the streamer fraction of the chamber signal as a function of $\mathrm{HV}_{\mathrm{ef} f}$.

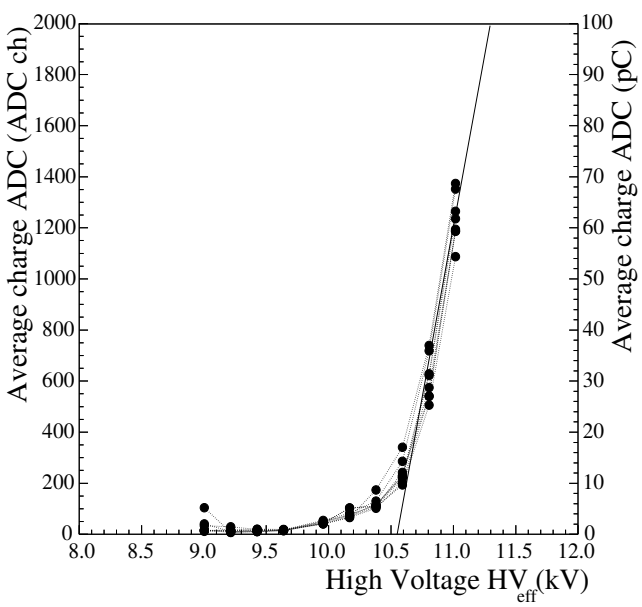

Figure 4: Avarege avalanche charge of the eight monitor chamber signal as a function of $\mathrm{HV}_{\text {ef } \mathrm{f}}$. The slope is about $25 \mathrm{ADC} \mathrm{ch} / 10$ or $1.2 \mathrm{pC} / 10 \mathrm{~V}$. Each point corresponds to 10000 triggers. 


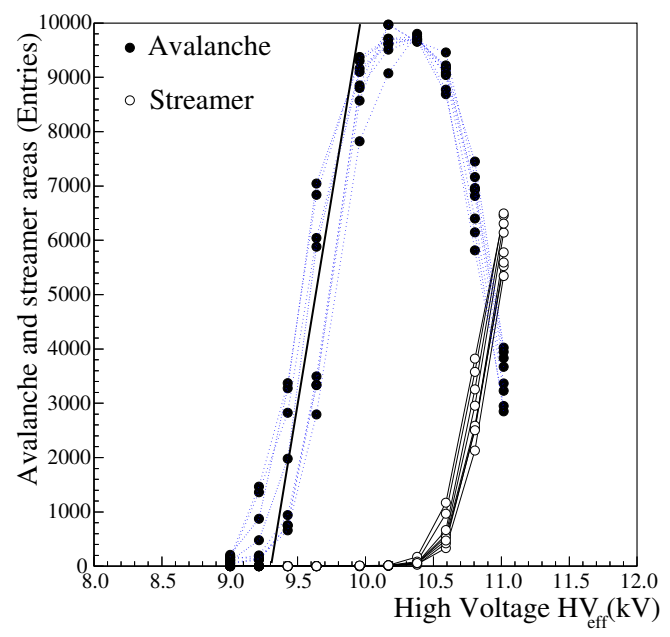

Figure 5: Streamer and avalanche yields as a function of $\mathrm{HV}_{\mathrm{ef} f}$. Each point corresponds to 10000 collected triggers. The solid line has a slope of approximately 130 events/10 V corresponding to a sensitivity of $1.3 \% / 10 \mathrm{~V}$.

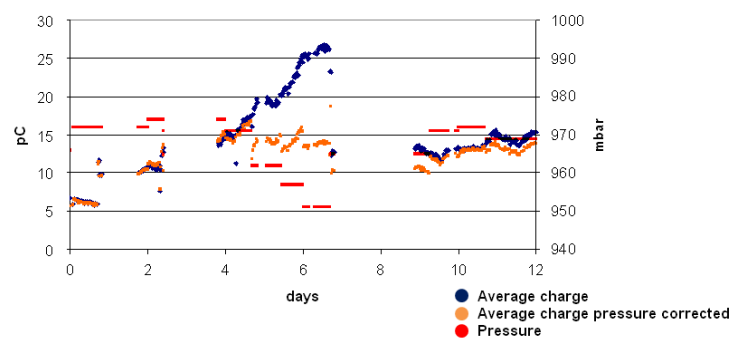

Figure 6: Average charge and pressure-corrected charge

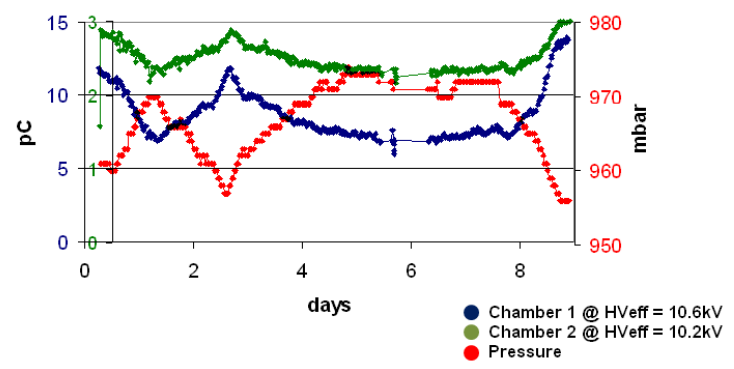

Figure 7: Average charge of two chambers at different voltages as influenced by pressure 


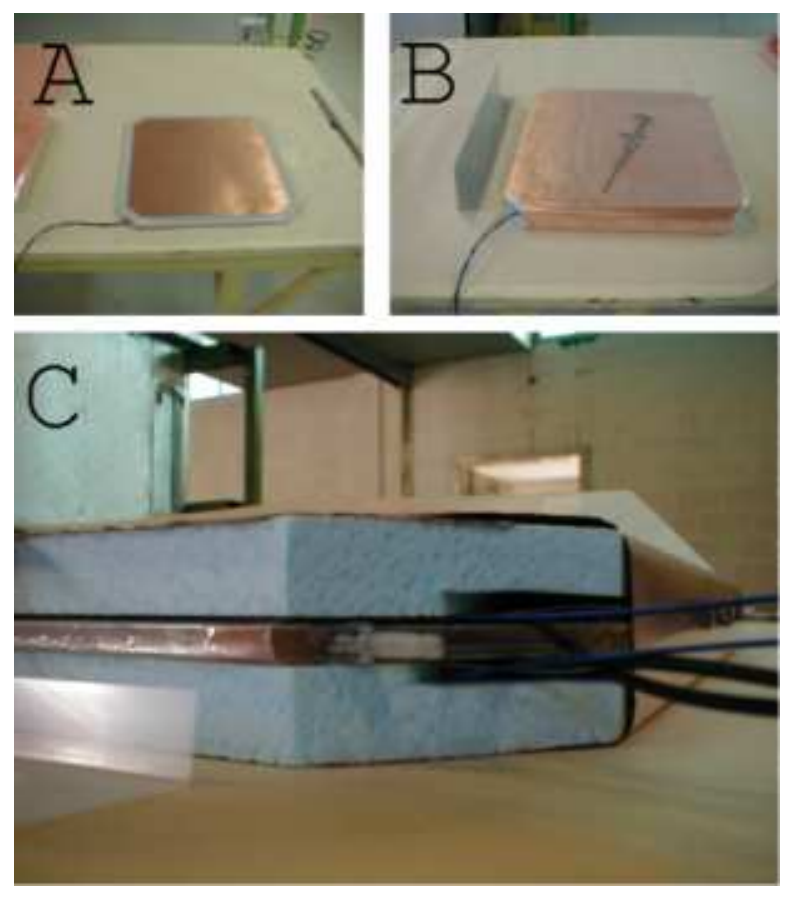




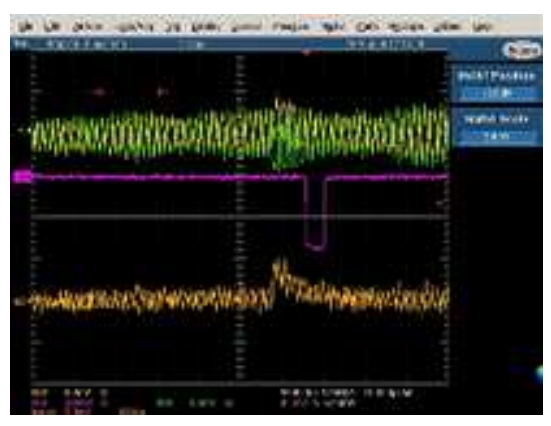




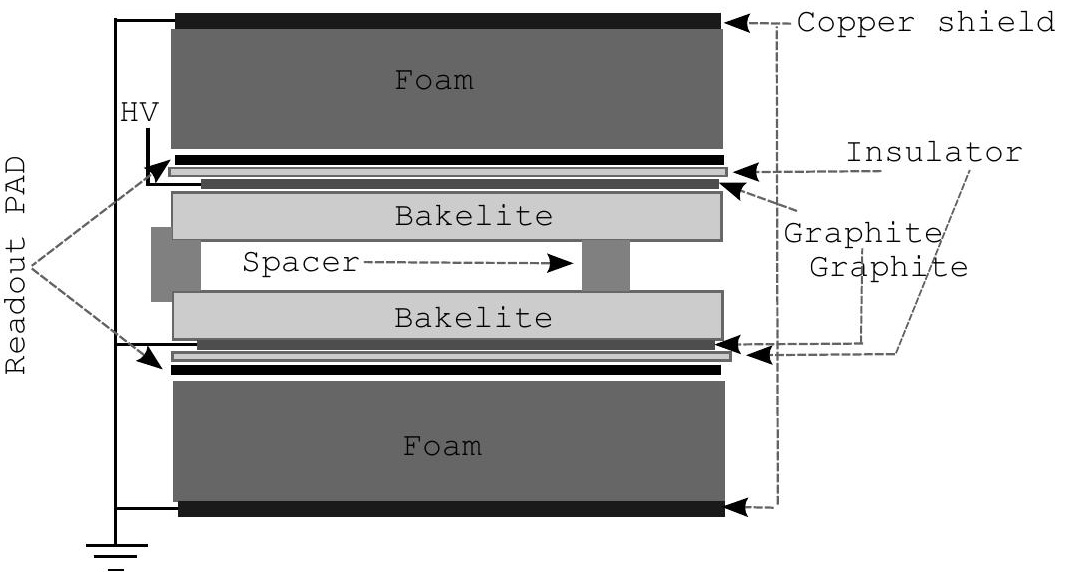




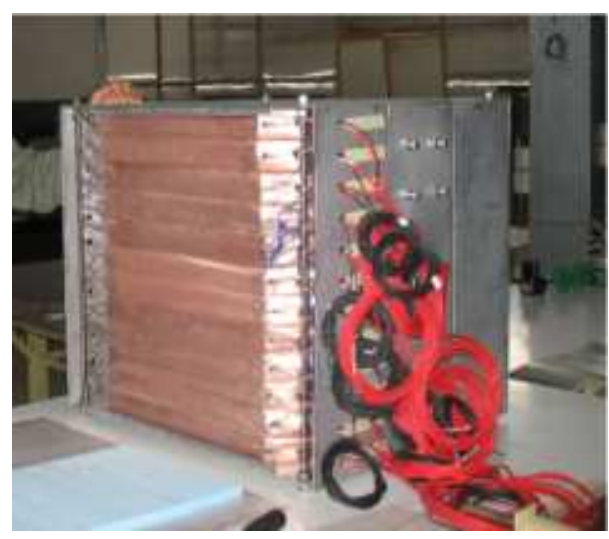

